Article

\title{
Simulated Microgravity Increases the Permeability of HUVEC Monolayer through Up-Regulation of Rap1GAP and Decreased Rap2 Activation
}

\author{
Shuliang Shi ${ }^{1}$, Jing $\mathrm{Li}^{1}$, Erzhuo Li ${ }^{1}$, Wenqi Guo ${ }^{1}$, Yao He ${ }^{1}$, Jinpeng Wang ${ }^{1}$, Yao Zhang ${ }^{1}$, Lei Yue ${ }^{1}$ \\ and Lijun Wei ${ }^{1,2, *}$
}

check for

updates

Citation: Shi, S.; Li, J.; Li, E.; Guo, W.; He, Y.; Wang, J.; Zhang, Y.; Yue, L.; Wei, L. Simulated Microgravity Increases the Permeability of HUVEC Monolayer through Up-Regulation of Rap1GAP and Decreased Rap2 Activation. Int. J. Mol. Sci. 2022, 23, 630. https://doi.org/10.3390/ ijms23020630

Academic Editor: Johann Bauer

Received: 1 September 2021 Accepted: 30 December 2021

Published: 6 January 2022

Publisher's Note: MDPI stays neutral with regard to jurisdictional claims in published maps and institutional affiliations.

Copyright: (C) 2022 by the authors. Licensee MDPI, Basel, Switzerland. This article is an open access article distributed under the terms and conditions of the Creative Commons Attribution (CC BY) license (https:// creativecommons.org/licenses/by/ $4.0 /)$.
1 School of Life Science and Technology, Harbin Institute of Technology, Harbin 150001, China; liangss@hit.edu.cn (S.S.); yangqianusst@163.com (J.L.); s1005674406@163.com (E.L.); guowenqi0304@163.com (W.G.); hy17861120139@163.com (Y.H.); 20B928024@stu.hit.edu.cn (J.W.); zhangyao83326@163.com (Y.Z.); yuelei@hit.edu.cn (L.Y.)

2 State Key Laboratory of Space Medicine Fundamentals and Application, Chinese Astronaut Research and Training Center, Beijing 100094, China

* Correspondence: weilijun@hit.edu.cn; Tel.: +86-156-3604-9367

\begin{abstract}
Space microgravity condition has great physiological influence on astronauts' health. The interaction of endothelial cells, which control vascular permeability and immune responses, is sensitive to mechanical stress. However, whether microgravity has significant effects on the physiological function of the endothelium has not been investigated. In order to address such a question, a clinostat-based culture model with a HUVEC monolayer being inside the culture vessel under the simulated microgravity (SMG) was established. The transmittance of FITC-tagged dextran was used to estimate the change of integrity of the adherens junction of the HUVEC monolayer. Firstly, we found that the permeability of the HUVEC monolayer was largely increased after SMG treatment. To elucidate the mechanism of the increased permeability of the HUVEC monolayer under SMG, the levels of total expression and activated protein levels of Rap1 and Rap2 in HUVEC cells, which regulate the adherens junction of endothelial cells, were detected by WB and GST pull-down after SMG. As the activation of both Rap1 and Rap2 was significantly decreased under SMG, the expression of Rap1GEF1 (C3G) and Rap1GAP in HUVECs, which regulate the activation of them, was further determined. The results indicate that both C3G and Rap1GAP showed a time-dependent increase with the expression of Rap1GAP being dominant at $48 \mathrm{~h}$ after SMG. The down-regulation of the expression of junctional proteins, VE-cadherin and $\beta$-catenin, in HUVEC cells was also confirmed by WB and immunofluorescence after SMG. To clarify whether up-regulation of Rap1GAP is necessary for the increased permeability of the HUVEC monolayer after SMG, the expression of Rap1GAP was knocked down by Rap1GAP-shRNA, and the change of permeability of the HUVEC monolayer was detected. The results indicate that knock-down of Rap1GAP reduced SMG-induced leaking of the HUVEC monolayer in a time-dependent manner. In total, our results indicate that the Rap1GAP-Rap signal axis was necessary for the increased permeability of the HUVEC monolayer along with the down-regulation of junctional molecules including VE-cadherin and $\beta$-catenin.
\end{abstract}

Keywords: simulated microgravity; adhesions junction; HUVEC cell; Rap1GAP; VE-cadherin

\section{Introduction}

The endothelium formed by an endothelial cell monolayer functions as a selective barrier between the bloodstream and tissues, preventing the leaking of small molecules, macromolecules, and cells from blood to the underlying tissue [1,2]. The significance of vascular integrity controlled by endothelial cell junctions is stressed by the fact that vascular hyperpermeability is related to diseases such as hemorrhage, chronic atherosclerosis, inflammation, and diabetes [3]. Adherens junctions are highly dynamic, and the exchange 
of molecules or extravasation and intravasation of white blood cells across the endothelial barrier could be achieved by regulation of the permeability of the endothelium [4]. The opening or closing of the endothelial barrier, which is dependent on the dynamics of adherens junctions, are regulated by the perivascular microenvironment, such as angiogenic cues, shear stress, or inflammatory factors, etc.

Vascular endothelial cadherin (VE-cadherin), the transmembrane glycoprotein restricted in endothelial cells, plays a key role in the regulation of adherens junctions [5]. The trans-interaction of VE-cadherin between endothelial cells could recruit $\beta$-catenin and p120-catenin to the cytosolic domain of VE-cadherin to stabilize the endothelial adherens junctions. Furthermore, the interaction of $\alpha$-catenin, vinculin, and EPLIN with the cytosolic domain of VE-cadherin could mediate the connection of adherens junctions to the actin cytoskeleton to form zonula adherens and maintain the functional integrity of the vascular barrier of the endothelium by facilitating the formation of adherens junctions between endothelial cells [6-11]. The regulation of the permeability of endothelial adherens junctions involves a complex process that contains vesicular trafficking, junctional rearrangements, and accurate cytoskeletal dynamics [9]. The expression and localization of VE-cadherin stand the focal point of the permeability regulation, as it dictates the level of expression and the localization of other junctional molecules, including claudin-5, N-cadherin, and so on [10].

Availability of VE-cadherin at the plasma membrane is necessary for endothelial barrier function, as VE-cadherin internalization or down-regulation is associated with the loss of cell-cell junctions, leading to enhanced cell migration and permeability [12]. Small G proteins including Rho family members (RhoA, Cdc42, and Rac1) and Rap family molecules (Rap1, Rap2) play key roles in the regulation of the barrier function of VE-cadherin-mediated adherens junctions by working downstream of the perivascular microenvironment and mechanical stress. Hormones and agonists regulate the permeability of the endothelium through intracellular signaling events that modulate the strength of the HUVEC monolayer formed by VE-cadherin $[13,14]$. Meanwhile, several signaling pathways have been shown to regulate the endocytosis of VE-cadherin in clathrin-coated pits including PI3K, Src, and PAK which work together to modulate the adherens junctions formed between endothelial cells [15].

Rap subfamily small G proteins consisting of closely related members, Rap1 and Rap2, belong to the Ras superfamily. Rap proteins function as a molecular switch that shifts between GDP-bound inactivated state and GTP-bound activated state, depending on the availability of two groups of factors: Rap guanine nucleotide factors (RapGEFs) and Rap GTPase-activating proteins (RapGAP), such as other G proteins [16].

Multiple guanine nucleotide exchange factors for small GTPase Rap1 and Rap2 mediate the signaling that regulates the tightening of the endothelium [17]. Among them, C3G, also named Rap1GEF1, exchange proteins activated by cAMP (Epac) and PDZ-GEF, have been proposed to regulate the formation of adherens junctions between endothelial cells through the activation of Rap1 [17,18]. In addition to its role in the activation of PKA, cAMP is a direct activator of Epac1 and Epac2 which activate both Rap1 and Rap2 [19]. C3G and PDZ-GEF could activate both Rap1 and Rap2, whereas RasGEF1 shows specificity toward Rap2 [20,21]. Epac1 decreases the cell permeability through its guanine nucleotide exchange factor (GEF) activity toward Rap1 and also through direct effects on microtubules [22]. C3G, the first found guanine nucleotide exchange factor for Rap1, is necessary for the recovery of the EC barrier through activating Rap1, following the thrombin-induced increases of endothelial permeability $[23,24]$.

Rap1 (Krev-1), originally identified as a gene with revertant-inducing activity on K-Ras oncogene, exists as two isoforms, Rap1A and Rap1B, regulating a variety of cellular functions including cell transformation, cell spreading, and formation of adherens junction between cells [25-30]. Rap1 was also isolated in a search for homologous genes to Drosophila Dras 3 along with Rap2 [31]. Rap1GAP, firstly isolated as a specific GTPaseactivating protein toward Rap1 (Krev-1), negatively regulates the activation of Rap1 and 
Rap2 by promoting the intrinsic GTPase activity that catalyzes the degradation of GTP bound to Rap proteins to GDP [21,32]. The overexpression of Rap1GAPs leads to an increase in endothelial permeability by abolishing Rap1 activity [29,30]. Deletion of Rap1GAP, in fact, prevents the formation of adherens junctions between carcinoma cells [33]. Although Rap2 shares a 65\% homology in amino acid sequence with Rap1, the function in the regulation of the endothelial permeability of Rap2 seems not to be consistent with Rap1 [34]. There are three isoforms of Rap2, Rap2A, Rap2B, and Rap2C, showing the major differences at the $\mathrm{C}$-terminal part of the proteins, which determines different subcellular localization of them [35]. Rap2-specific effectors, including MAP4K4, MINK, TINK, and PARG, may connect Rap2 signaling to the regulation of the microfilament cytoskeleton [36].

The endothelial cells are very sensitive to microgravity and would show morphological and functional changes under a microgravity condition $[37,38]$. To explore whether the physiological function of the endothelium could be altered during microgravity conditions, we established a clinostat-based HUVEC monolayer permeability assay model under SMG, and the permeability of the HUVEC monolayer was detected under SMG. The physiological impact on the HUVEC monolayer of small G protein Rap1 and Rap2, as well as their upstream regulators including Rap1GAP and C3G, were investigated.

\section{Results}

\subsection{Modulation of Endothelium Permeability by Simulated Microgravity}

The phenotype of HUVECs, human umbilical vein endothelial cells, has shown to be highly affected by microgravity leading to various physiological changes including an abnormal cytoskeleton and downregulated mechanic sensing proteins [38]. However, whether microgravity has effects on the physiological function of the endothelium has not been reported up until now. To investigate the potential effects of microgravity on the permeability of the endothelium, we established a monolayer HUVEC cell model under SMG by inserting a transwell chamber with pores of $3 \mu \mathrm{m}$ in diameter and determined the effects of SMG on the permeability of monolayer HUVEC cells (Figure 1).

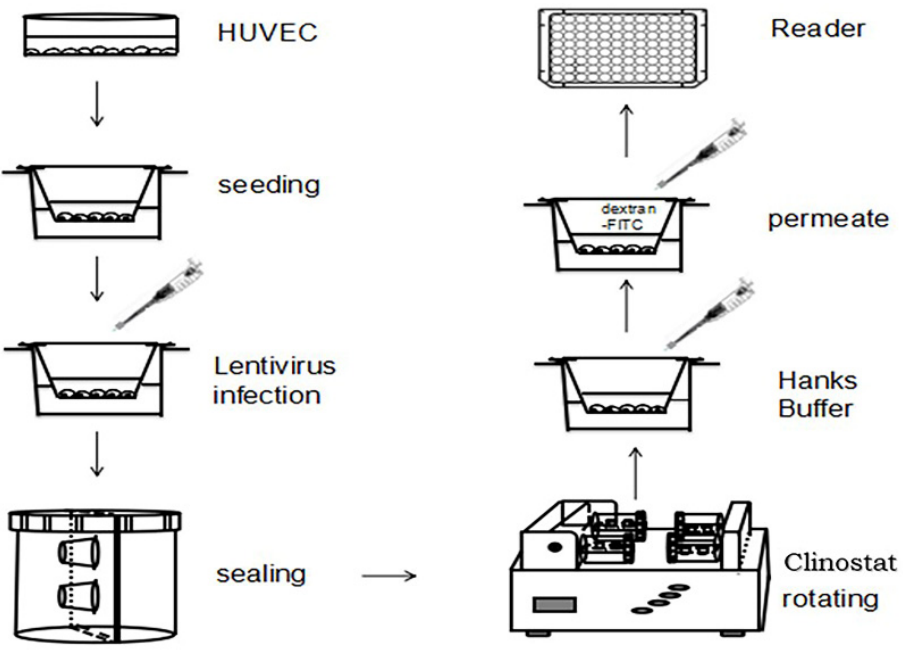

Figure 1. Establishment of monolayer cell permeability model under SMG.

The results show that the permeability of the HUVEC monolayer was increased at $24 \mathrm{~h}$ SMG treatment and recovered to the normal level gradually until 48h after SMG (Figure 2). The increased permeability of the HUVEC monolayer suggested that the adherens junction of HUVEC cells might be decreased by SMG treatment. 


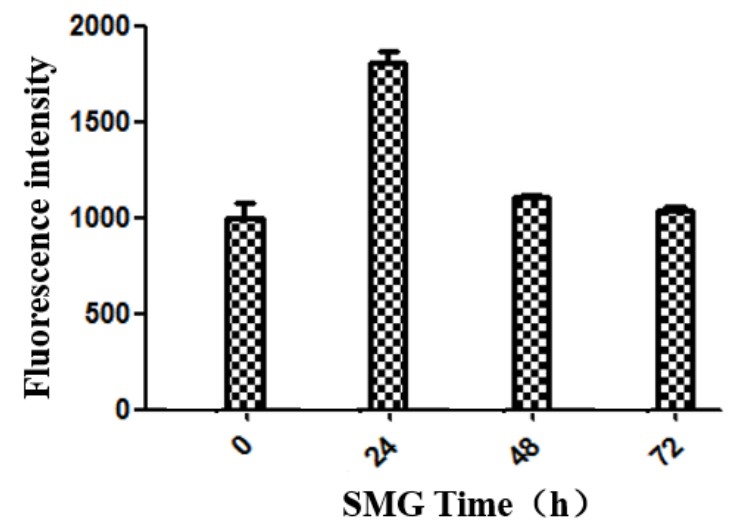

Figure 2. Permeability of HUVEC monolayer under SMG. HUVEC cells were plated on culture chamber and grown to confluence to form monolayer and set to 2D clinorotation. Then the permeability of HUVEC monolayer under SMG for 24, 48, and $72 \mathrm{~h}$ was assayed.

\subsection{The Activation of Rap2 Was Down-Regulated under SMG}

Rap small G proteins regulate the formation of endothelial cell adherens junctions [17]. To elucidate the mechanism of enhanced permeability of the HUVEC monolayer under SMG, the activity of both Rap1 and Rap2 was detected after SMG using a GST pull-down experiment. The activation of Rap1 was slightly decreased, while Rap2 activation was largely down-regulated with the lowest activation at $48 \mathrm{~h}$ treatment (Figures 3 and 4 ). The activation levels of Rap1 and Rap2 were slightly recovered at $72 \mathrm{~h}$ treatment. However, the activation level of Rap2 in HUVEC cells under SMG was still greatly lower than the level of control. On the other hand, the level of expression of Rap1 and Rap2 showed a similar pattern with a slight increase at $24 \mathrm{~h}$ and a gradual recovery since $48 \mathrm{~h}$ SMG treatment.

(a)

$$
\text { CK } \frac{\text { SMG }}{24 \mathrm{~h} \quad 48 \mathrm{~h} 72 \mathrm{~h}}
$$

\section{Rap2-GTP}

Total Rap2

\section{GAPDH}

(b)

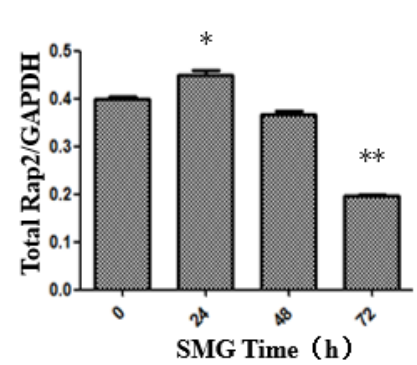

(c)

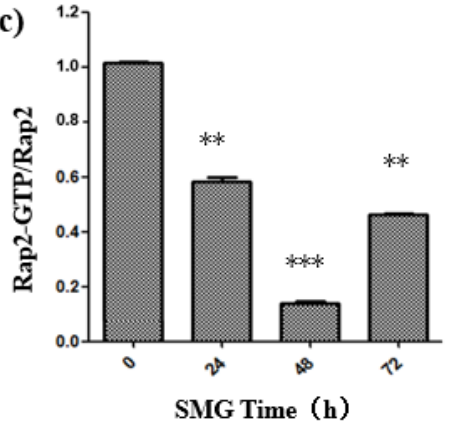

Figure 3. The activation of Rap2 was down-regulated by SMG. (a) Expression and activation of Rap2 after different times of SMG treatment were detected by Western blot and GST pull-down assay, respectively. (b) The relative Rap2 expression under SMG for 24, 48, and $72 \mathrm{~h}$ was calculated based on panel (a). (c) The relative activation of Rap2 under SMG for 24, 48, and $72 \mathrm{~h}$ was calculated based on panel (a). The statistical results shown represent the means $(n=3)$ vs. the control group $(n=3)$; ${ }^{*} p<0.05,{ }^{* *} p<0.01,{ }^{* * *} p<0.001$. 
(a)

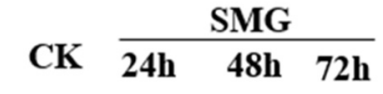

Rap1-GTP

Total Rap1

GAPDH

(b)

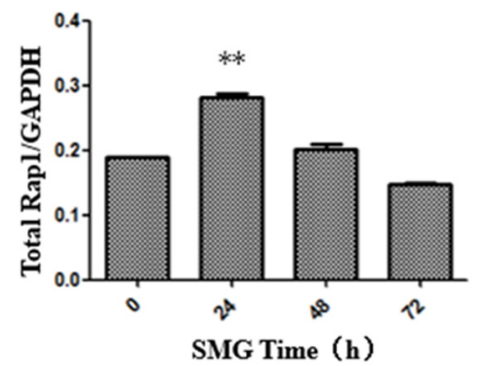

(c)

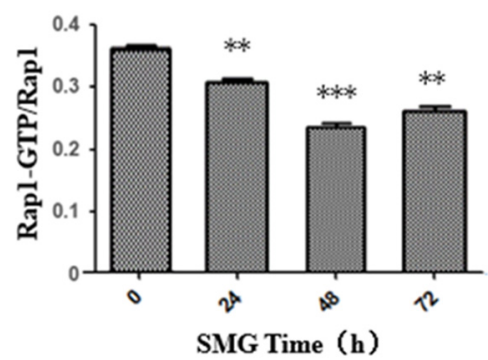

Figure 4. The activation of Rap1 was down-regulated by SMG. Expression and activation of Rap1 protein under SMG for 24, 48, and $72 \mathrm{~h}$ were detected by Western blot and GST pull-down assay, respectively. (b) The relative Rap1 expression under SMG for 24, 48, and $72 \mathrm{~h}$ was calculated based on panel (a). (c) The relative activation of Rap2 under SMG for 24, 48, and $72 \mathrm{~h}$ was determined based on panel (a). The statistical results shown represent the means $(n=3)$ vs. the control group $(n=3)$; ** $p<0.01,{ }^{* * *} p<0.001$.

\subsection{Rap1GAP Was Down-Regulated under Simulated Microgravity}

RapGEF1 or C3G, which is short for rap guanine nucleotide exchange factor 1, could up-regulate the activation of Rap1 and Rap2. Rap1GAPs down-regulate the activation of Rap1 and Rap2 [21]. To elucidate the mechanism of down-regulation of Rap2, both the activation of RapGEF1 and Rap1GAP were determined under microgravity. The results indicate that both C3G and Rap1GAP were up-regulated, but the pattern and intensity of their expression differed greatly (Figure 5). The expression of C3G reached its peak at $24 \mathrm{~h}$, and C3G was up-regulated by $60 \%$ at that time under SMG. Meanwhile, the expression of Rap1GAP peaked at $48 \mathrm{~h}$, and C3G was up-regulated by five times at that time under SMG. Comparing the expression pattern and intensity of C3G and Rap1GAP, the enhanced level of Rap1GAP should be dominant over C3G to regulate the activation of Rap1 and Rap2 at $24 \mathrm{~h}$ and $48 \mathrm{~h}$ treatment of SMG.

\subsection{The Expression of VE-Cadherin and $\beta$-Catenin Was Down-Regulated under Simulated Microgravity}

As the increased permeability of the HUVEC monolayer may attribute to the altered expression or subcellular localization of VE-cadherin, the expression and distribution of VE-cadherin were detected by Western blotting and immunofluorescence assay after SMG (Figures 6 and 7). As expected, the expression of VE-cadherin was significantly downregulated with the lowest expression of VE-cadherin at $24 \mathrm{~h}$ after SMG. The expression of VE-cadherin was gradually recovered from $48 \mathrm{~h}$ to $72 \mathrm{~h}$ under SMG (Figure 6). The down-regulated expression of VE-cadherin was also confirmed by immunofluorescence using anti-VE-cadherin specific antibody (Figure 7). However, we could not find the change in the subcellular distribution pattern of VE-cadherin. Besides the membrane localization at cell-cell contact, VE-cadherin could be detected at the perinuclear region and cytosol of HUVEC cells both in control and SMG treatments. As $\beta$-catenin connects VE-cadherin to actin filaments through interaction with the intracellular domain of VEcadherin and regulates the adherens junction formation between endothelial cells, we tested whether the expression and distribution of $\beta$-catenin were affected by SMG treatment using Western blotting and immunofluorescence assay with anti- $\beta$-catenin specific antibody 
(Figures 6 and 7). The results indicate that, similar to VE-cadherin, the expression of $\beta$-catenin showed a pattern of an upward curve with the valley of VE-cadherin at $24 \mathrm{~h}$ SMG treatment. However, the extent of down-regulation of $\beta$-catenin by SMG was much higher than VE-cadherin. The expression of $\beta$-catenin was gradually recovered from $48 \mathrm{~h}$ and peaked at $72 \mathrm{~h}$ after SMG (Figure 6). The down-regulated expression of $\beta$-catenin was also confirmed by immunofluorescence using anti- $\beta$-catenin specific antibody (Figure 7). Although $\beta$-catenin mainly showed a membrane-localized pattern, the level of fluorescence intensity was much lower at $24 \mathrm{~h} \mathrm{SMG}$ than that in the control cells.

(a)
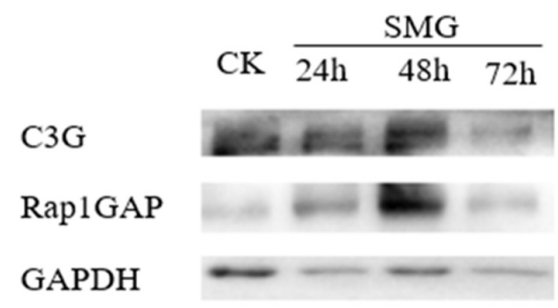

(b)

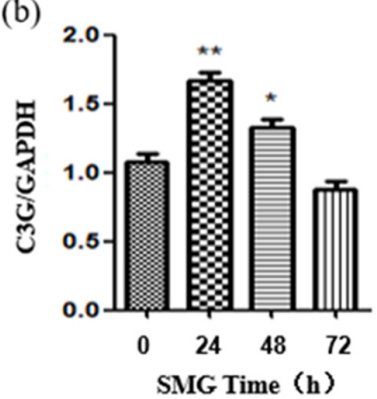

(c)

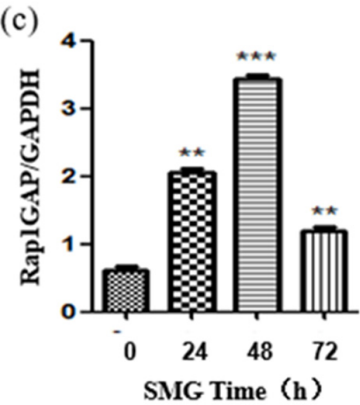

Figure 5. The expression of C3G and Rap1GAP protein under SMG was up-regulated. (a) The proteins of HUVEC cells were extracted after SMG treatment, and the expression of C3G and Rap1GAP were detected with Western blots using anti-C3G or anti-Rap1GAP specific antibody, respectively. $(\mathbf{b}, \mathbf{c})$ The relative expression of C3G and Rap1GAP under SMG for 24, 48, and $72 \mathrm{~h}$ was calculated based on intense assay of each band of protein. The statistical results shown represent the means $(n=3)$ vs. the control group $(n=3) ;{ }^{*} p<0.05,{ }^{* *} p<0.01,{ }^{* * *} p<0.001$.

(a)

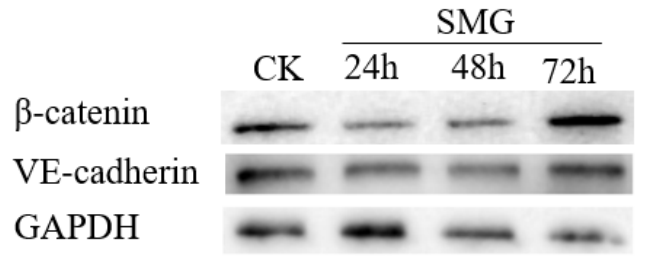

(b)
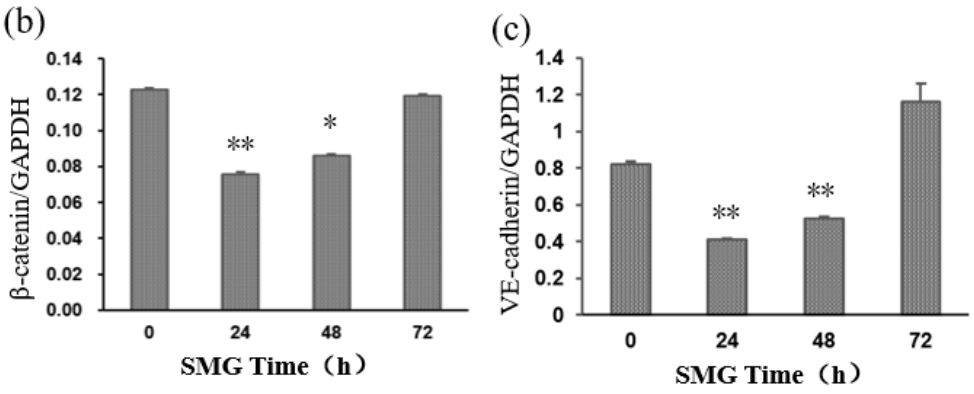

Figure 6. The expression of VE-cadherin and $\beta$-catenin was down-regulated under SMG. (a)The total proteins were isolated from HUVEC cells treated with SMG, and expression of $\beta$-catenin and VE-cadherin protein was detected by Western blot with anti- $\beta$-catenin or anti-VE-cadherin antibody, respectively. (b,c) The relative expression of $\beta$-catenin and VE-cadherin under SMG for 24,48 , and $72 \mathrm{~h}$ was calculated based on intense assay of each band of protein. The statistical results shown represent the means $(n=3)$ vs. the control group $(n=3) ;^{*} p<0.05,{ }^{* *} p<0.01$. 


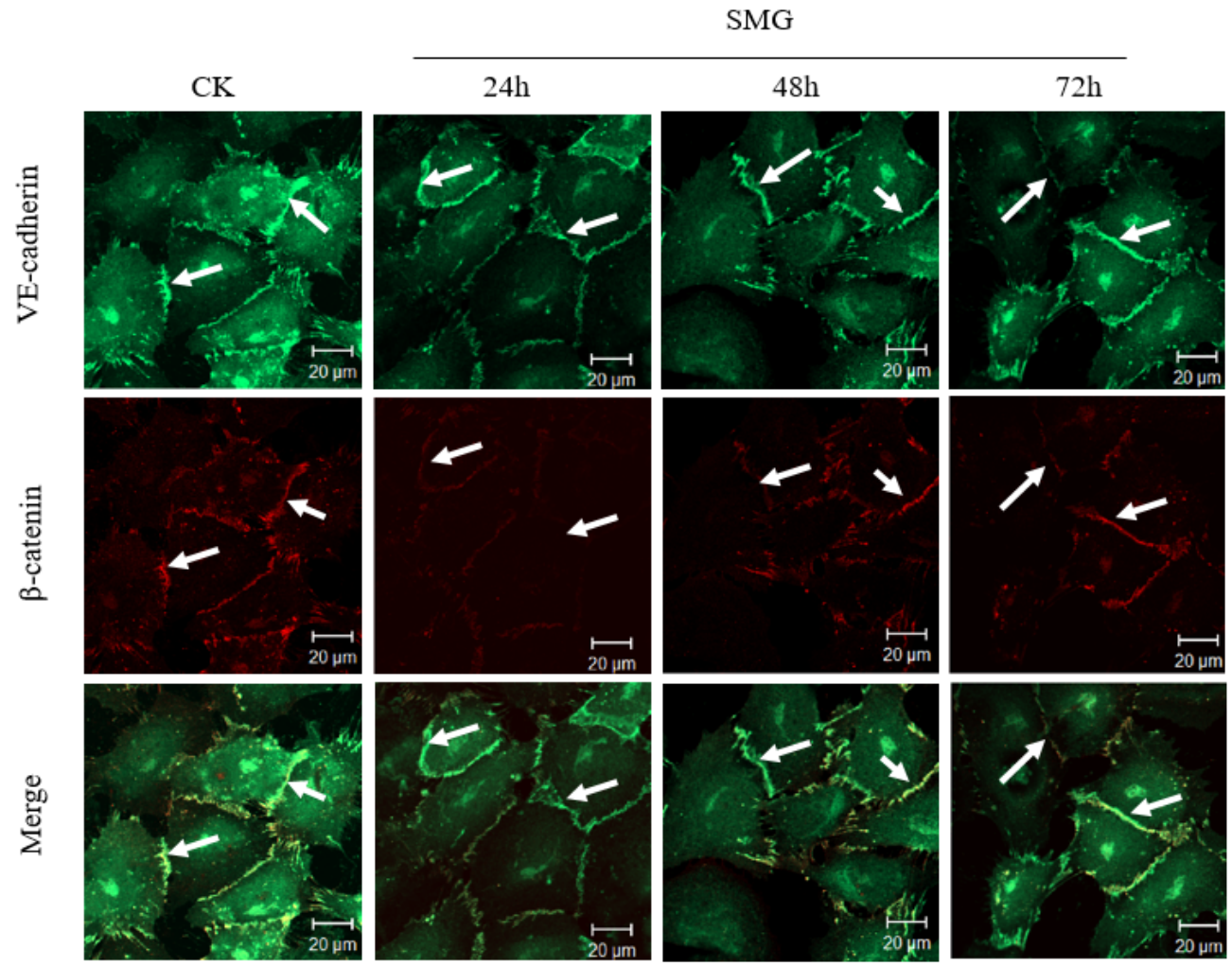

Figure 7. The expression and distribution of VE-cadherin and $\beta$-catenin in HUVEC cells under SMG. The upper panel: VE-cadherin in green; the middle panel: $\beta$-catenin in red; the lower panel: merged. The white arrows shows the location of VE-cadherin (first line), $\beta$-catenin (second line) and the co-localization of VE-cadherin and $\beta$-catenin. Scale bar $=20 \mu \mathrm{m}$.

\subsection{The Distribution of Actin Filaments Was Modulated by Simulated Microgravity}

A cortical distribution of actin filaments under the cell-cell adherens junction forms through binding to the intracellular domain of VE-cadherin mediated by $\alpha$-catenin, $\beta$ catenin, and p120-catenin and enhances the cell-cell interaction which contributes to decreased permeability through the endothelial cell layer. To detect whether SMG could affect the cortical distribution of actin filaments, immunofluorescence experiments were performed after SMG treatment of HUVEC cells using phalloidin staining (Figure 8). In contrast to the major cortical distribution of actin filaments under the cell-cell junctions in control HUVEC cells, the cortical distribution of actin filaments under cell-cell junctions was decreased. Instead, the stress fiber formation at the region of the cell bottom was apparent at $24 \mathrm{~h}$ and could still be seen until $72 \mathrm{~h} \mathrm{SMG}$ treatment. These results suggest that the shift of cortical distribution to the stress fiber of actin filaments may be involved in the increased permeability through the HUVEC monolayer under SMG.
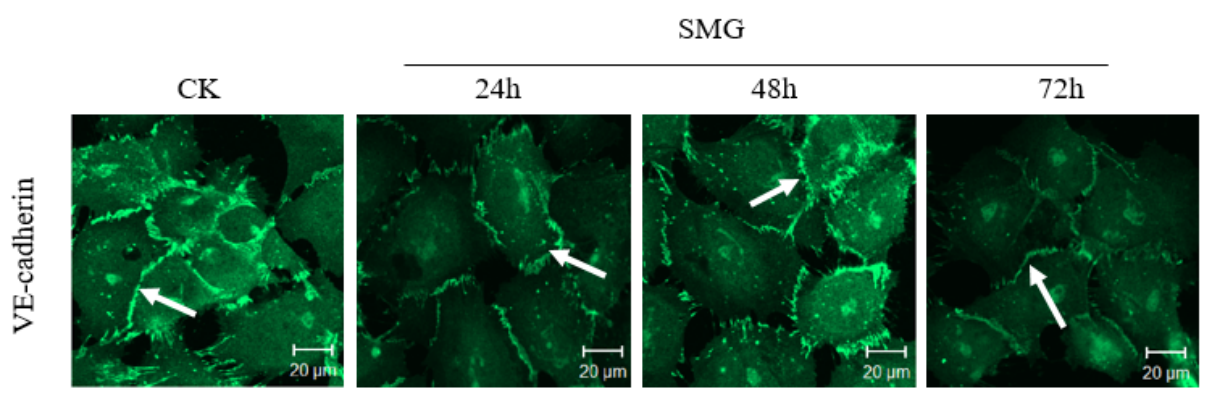

Figure 8. Cont. 

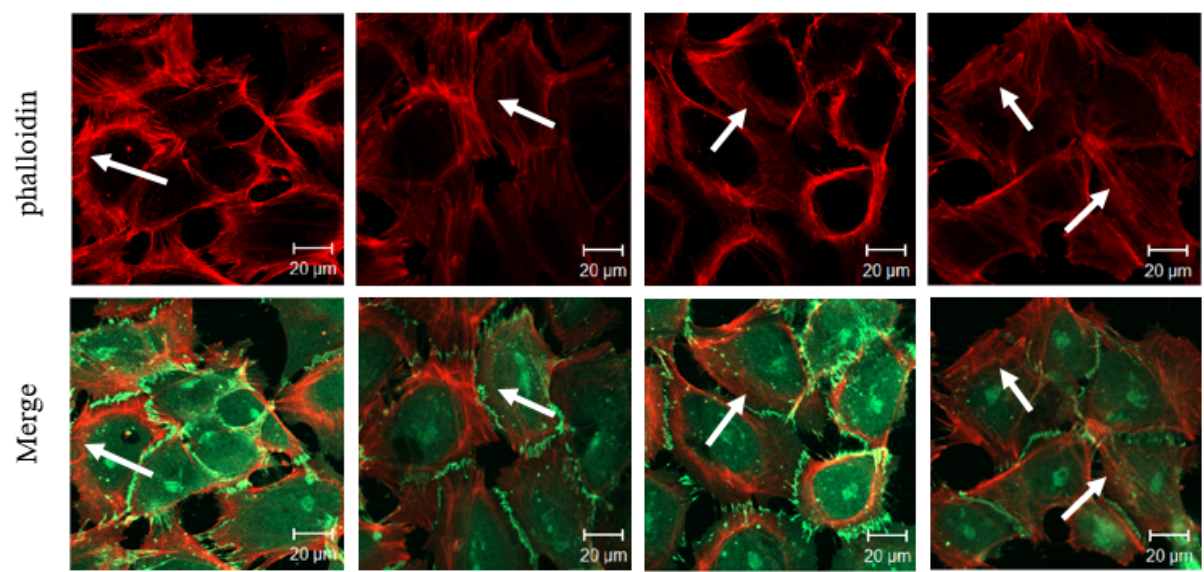

Figure 8. The expression and distribution of VE-cadherin and actin filaments in HUVEC cells under SMG. The upper panel: VE-cadherin stained in green with antibody against VE-cadherin; middle panel: actin filaments stained in red with phalloidin conjugated with Texas Red; the lower panel: merged. The white arrows in the first line show the localization of VE-cadherin. The white arrows in the second line show the localization of F-acting. The white arrows in the third line show F-acting localized beneath adherens junctions formed by VE-cadherin. Scale bar $=20 \mu \mathrm{m}$.

\subsection{The Knock-Down of Rap1GAP Decreased the Permeability of HUVEC Monolayer under Simulated Microgravity}

It has been reported that Rap2 signaling is activated during cell-cell junction formation and may be involved in the regulation of permeability of the endothelial cell monolayer. Rap1 GAP negatively regulates the activation of Rap2 by promoting the shift of the GTPbinding state to the GDP-binding state of Rap2 [20]. To address the role of Rap1GAP in the permeability of the HUVEC monolayer, Rap1GAPs were knocked down through shRNA expression. The permeability assay of monolayer HUVEC cells expressing shRNA specific to Rap1GAP was performed using a clinostat-based model under SMG (Figure 9). In contrast to the up-regulation of permeability of the HUVEC monolayer under the control condition, the knock-down of Rap1GAP decreased the permeability of the HUVEC monolayer under SMG, and the decreased permeability lasted until $72 \mathrm{~h}$ after SMG treatment. The results indicate that Rap1GAP could regulate the permeability of the HUVEC monolayer under SMG.

(a)

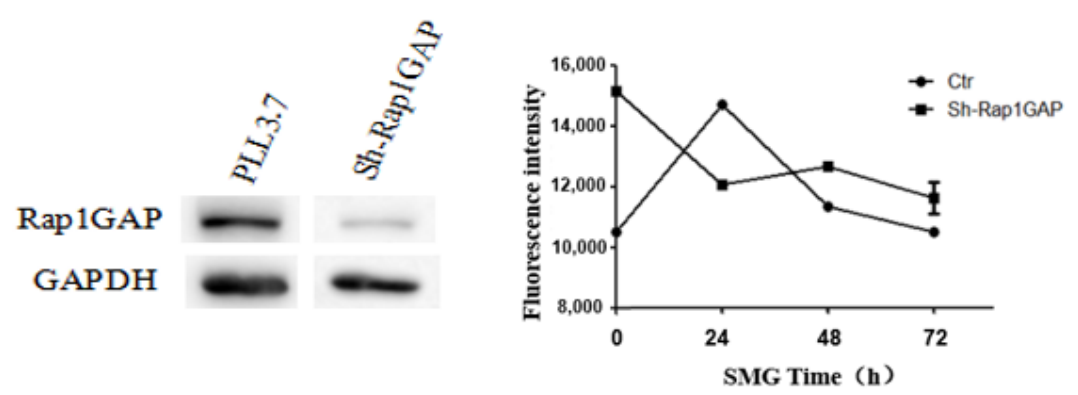

Figure 9. Rap1GAP interfering with cell line construction and detection. (a) Intracellular Rap1GAP interference efficiency. (b) The effect of simulated microgravity on the permeability of monolayer HUVEC cells after interfered with by Rap1GAP.

\section{Discussion}

It has been shown that microgravity has a variety of biological effects including an abnormal cytoskeleton and down-regulated mechanic sensing proteins on endothelial 
cells [38-42]. However, whether microgravity has effects on the physiological functions of the endothelium has not been addressed up until now.

HUVECs are human umbilical vein endothelial cells isolated from the endothelium of umbilical veins. They are well used to investigate the phenotype of the cell-cell junctions and trans-endothelium permeability for macromolecules and blood cells [33]. To address the physiological effects of SMG on the integrity of the endothelium, we established a HUVEC monolayer model based on 2D-clinostat rotation to monitor the change of permeability of the HUVEC monolayer under SMG. We found that the SMG largely increased the permeability of the HUVEC monolayer in a time-dependent manner. In addition, with the extension of SMG treatment, the permeability of the HUVEC monolayer recovered to the normal level at 48 and $72 \mathrm{~h}$. Robert Szulcek et al. challenged an assay for the barrier integrity of the HUVEC monolayer under RPM-based microgravity or large-diameter centrifuge (LDC)-based hyper-gravity and showed that simulated microgravity leads to a decreased barrier integrity of the HUVEC monolayer, although the mechanism for the change in endothelial barrier integrity has not been investigated [39].

It has been reported that Rap1 and Rap2 antagonistically regulate the integrity of adherens junction between endothelial cells. Rap1 promoted the barrier resistance of the HUVEC monolayer, which was inhibited by Rap2 by an electrical cell impedance sensing (ECIS) assay [39]. In response to microgravity stress, in HUVEC cells, the expression of a large number of genes is drastically changed, which are mainly involved in cell adhesion, cell apoptosis, and cell cycle processes [41]. In addition, SMG affects the complexity of intracellular skeletal proteins and induces cytoskeletal rearrangement [42]. These findings suggest that Rap1 and Rap2 may respond to the simulated microgravity and thus regulate the adherens junctions between monolayer HUVEC cells under microgravity.

In this study, we found that the expression level of Rap1 and Rap2 proteins in HUVEC cells increased after $24 \mathrm{~h}$ of SMG treatment but decreased gradually to the basal level or to an even lower level than the control with regard to Rap2. On the contrary, the activation of Rap1 and Rap2 under SMG treatment decreased significantly, reaching the lowest value at $48 \mathrm{~h}$, and then recovered partially. The decline rate of Rap2 reached $80 \%$, which was much higher than the $35 \%$ decrease of Rap1 activation, suggesting that Rap2 plays a major biological role in response to SMG stress. Although there is still no direct evidence to support that Rap2 signaling could positively regulate the formation of adherens junctions up until now, the trans-dimerization of JAM-A, a tight-junction-associated protein, upregulates the activation of Rap2 and further participants in the positive regulation of cell adherens junction in HEK293 cells [43,44].

As a negative regulator for the activation of both Rap1 and Rap2, Rap1GAP downregulation in colon cancer cells inhibited the formation of adherens junction between cells but enhanced the adhesion of cells and extracellular matrix [45]. Therefore, the biological role of Rap1GAP should be dependent on the cell context and intercellular environment. Here, we found that the expression of Rap1GAP increased significantly in response to SMG treatment, peaking at $48 \mathrm{~h}$ after SMG. Meanwhile, the expression of C3G (also named Rap1GEF1) also showed a significant increase under SMG, with the highest level at $24 \mathrm{~h}$ SMG treatment. Meanwhile, it cannot be ignored that the expression level of Rap1GAP at $48 \mathrm{~h}$ SMG treatment increased five times compared to the control, which was much higher than the $50 \%$ increase of C3G at $24 \mathrm{~h}$ SMG treatment, suggesting that Rap1GAP plays a dominant role in regulating the activation of Rap2 under SMG treatment.

The formation of adherens junction is facilitated by the trans-interaction of VEcadherin between endothelial cells and formation of the cortical actin filament bundle beneath the plasma membrane through connecting to the intracellular domain of VE-cadherin through interaction with $\beta$-catenin which will strengthen the adherens junction [7,18]. In these results, the increased permeability of a monolayer of HUVECs under SMG should be related to the loosening of adherens between HUVEC cells. This result shows that the expression of VE-cadherin and $\beta$-catenin in HUVEC cells decreased significantly under SMG treatment, while their intracellular localization did not change significantly 
(Figures 6 and 7). Therefore, the increased permeability of the HUVEC monolayer resulted from SMG might just relate to the down-regulation of VE-cadherin and $\beta$-catenin in quantity but not the regulation of its intracellular distribution. However, our results do not rule out the mechanism of change at the posttranslational modification of VE-cadherin and $\beta$-catenin under SMG.

The increase in Rap1GAP expression under SMG is accompanied by significant downregulation of Rap1 and Rap2 activity, indicating that the Rap1GAP-Rap axis may play a key role in the integrity of the HUVEC monolayer. Although there were reports showing that both Rap1 and Rap2 could regulate adherens junction between endothelial cells, the reports regarding the role of Rap2 in adherens junction are still lacking and rather controversial [34,41]. In normal conditions, the permeability of monolayer HUVEC cells expressing Rap1GAPshRNA was higher than that of cells expressing scrambled shRNA (Figure 9). The importance of Rap1GAP in cell-cell adhesion is also reported in research on carcinoma cells: deletion of Rap1GAP prevented the formation of adherens junctions in colorectal carcinoma indicating that Rap1GAP is necessary for the formation of adherens junctions at a basal level $[32,46]$. However, compared to the cells expressing scrambled shRNA, the increase of permeability in the HUVEC monolayer induced by SMG was significantly inhibited by the interference of Rap1GAP, and this inhibition lasted until $72 \mathrm{~h}$ after SMG. To elucidate whether decreased activation of Rap1 or Rap2 could contribute to the increased permeability of the HUVEC monolayer under SMG, acting downstream of Rap1GAP, the activation of Rap1 and Rap2 in HUVEC cells expressing Rap1GAPshRNA or the control shRNA was detected after SMG (Figure S1). The activation of both Rap1 and Rap2 was similar to the grand level, indicating that the up-regulation of the permeability of the HUVEC monolayer by Rap1GAP under SMG was mediated by the down-regulation of Rap1 and Rap2.

In conclusion, by establishing a model to monitor the permeability of the HUVEC monolayer under SMG, we found that SMG significantly increased the permeability of the HUVEC monolayer. The mechanism underlying the up-regulated permeability may involve the small G protein Rap signaling pathway as the significant up-regulation of Rap1GAP and down-regulation of Rap2 and Rap1 were confirmed under SMG. Our findings provide new cues that connect the mechanic sensing signal to the increased permeability of the HUVEC monolayer through down-regulating cell-cell adherens junctions.

\section{Materials and Methods}

\subsection{Cultures of HUVEC}

HUVEC cell line was used for the present study and was purchased from ATCC. HUVEC cells were cultured in DMEM (Corning, Shanghai, China) with $20 \%(v / v)$ fetal calf serum (FBS, Gibco-BRL, Shanghai, China), supplemented with ECGS (MACGENE, Shanghai, China).

\subsection{Cell Culture in Rotating Culture Vessel}

Rotating wall vessel (2D-RWVS) was originally developed by China Astronaut Research and Training Center. HUVEC cells were seeded over a cover slip $(226 \times 266 \times 0.5 \mathrm{~mm})$ and cultured on the slip for $24 \mathrm{~h}$. The slip was then transferred into culture vessel of $0.04 \mathrm{~m}$ in diameter filled with growth medium. According to equipment manual and reference, the samples were rotated around the horizontal axis at $30 \mathrm{rpm}$ to simulate microgravity effect (SMG) $[47,48]$. The cells cultured in the RWVS vessel without rotation were considered as the untreated control (static control) group.

To avoid the possibility that changes in protein expression over time might affect the results of the experiment, our experiment was designed as follows:

After the cells were seeded over a cover slip, the simulated microgravity experiments were conducted after 24th hour, 48th hour, and 72nd hour, respectively, and those who had not been simulated-microgravity-treated were control group. Samples of each group were collected at $96 \mathrm{~h}$, and materials with microgravity for $72,48,24$, and $0 \mathrm{~h}$ (ground control) 
were obtained. Because the culture time of each group was $96 \mathrm{~h}$, the difference in protein expression was avoided.

\subsection{Construction of Interference Vector}

The oligos used in experiment are listed in Table 1 as follows.

Table 1. Oligo sequences.

\begin{tabular}{cc}
\hline Gene Name & Primer Sequence \\
\hline Sh-Rap1GAP-s & 5'-TGCAATGTGGTATTTCAAGAGAATAGAAT $^{\prime}$ AGAACCGATCCACATTGCTTTTTC-3' \\
Sh-Rap1GAP-a & 5'-TCGAGAAAAAAGCAATGTGGATCGGTTCT \\
& ATTCTCTTGAAATAGAACCGATCCACATTGCA-3' \\
\hline
\end{tabular}

h-Rap1GAP-s and Sh-Rap1GAP-a were mixed in annealing buffer and annealed by heating to $95^{\circ} \mathrm{C}$ for $5 \mathrm{~min}$, followed by a slow cooling to RT. pLentilox3.7Rap1GAPshRNA vector was constructed by inserting the annealed oligos to the HpaI-digested site of pLentilox3.7.

\subsection{Virus Packaging and Infection}

293T cells were grown to sub-confluence with DMEM medium supplemented with 10\% FBS and cotransfected by pLentilox3.7shRap1GAP or Lentilox3.7-scramble-shRNA, pCMVVSV-G), and pCAG-dR8.9. Forty-eight hours after transfection, the culture supernate was collected and filtered with filter of $0.45 \mu \mathrm{m}$ to prepare the virus suspension. Then, HUVEC cells were grown to $60 \%$ confluency in 12 -well plates and infected with the premixed virus suspension, M199 medium, and polybrene at a rate of 500:250:0.5. The culture suspension was replaced with fresh M199 medium supplemented with 20\% FBS and ECGS 24 h after infection.

\subsection{Screening of HUVEC Cells Expressing shRNAs}

HUVEC cells expressing Rap1GAPshRNA or scrambled shRNA were collected and diluted $48 \mathrm{~h}$ after infections to a 96-well plate to ensure that the number of cells in each well was 1-2 cells. When the single clone grew to $50 \%$ confluence, the cells were replated to 24-well plate and grown to confluence. Then the expression of Rap1GAP was detected by Western blot to determine the interference efficiency.

\subsection{HUVEC Monolayer Permeability Model}

A total of $4 \times 10^{4}$ HUVEC cells were seeded on a transwell chamber with pore of $3 \mu \mathrm{m}$ in diameter and allowed to grow until a uniform monolayer was formed by HUVEC cells. The chamber was then transferred to a culture vessel (Figure 1) containing M199 medium supplemented with $20 \%$ FBS and 0.5\% ECGS. The culture vessels set with HUVEC monolayer grown on the surface of filter of the chamber were set on 2D-clinostat and let to rotate horizontally to simulate the microgravity condition. The vessels set with HUVEC monolayer grown on the surface of filter of the chamber were set on 2D-clinostat and allowed to rotate vertically. The chambers were removed from the vessels 24,48 , and $72 \mathrm{~h}$ after rotation of vessels in $2 \mathrm{D}$-clinostat machine to a 24 -well plate. The medium in the upper room of the chamber was discarded, and the chamber was washed with Hanks' Buffer and added with $100 \mu \mathrm{L}$ of $2.5 \mathrm{mg} / \mathrm{mL}$ of FITC-dextran (MW4000, Sigma, Shanghai, China). The lower room of the chambers set on 24 -well plate was added with $500 \mu \mathrm{L} \mathrm{Hanks}^{\prime}$ Buffer. The chambers were incubated at $37^{\circ} \mathrm{C}$ with $5 \% \mathrm{CO}_{2}$ for $30 \mathrm{~min}$ in a dark condition. The chambers were removed from 24 -well plate, $50 \mu \mathrm{L}$ of solution in the lower room of the chamber was removed to 96-well plate, and the intensity of fluorescence for FITC in the lower room was measured in a microplate reader (Bio-Rad). The permeability assay of HUVEC monolayer was repeated three times for each time point. Meanwhile, the control experiment was performed by using the same protocol with different concentration of 
FITC-dextran (MW4000). The standard curve was plotted after monitoring intensity of fluorescence of the lower chamber at each concentration of FITC-dextran (Figure 1).

\subsection{Immunofluorescence Analysis}

HUVEC cells were fixed at room temperature with 4\% PFA (Sigma, St. Louis, MO, USA) for $15 \mathrm{~min}$, permeabilized in 1\% (v/v) TritonX-100 (AMERSCO, Washington, DC, USA) for $10 \mathrm{~min}$, and blocked with 5\% BSA (Sigma, St. Louis, MO, USA) for $60 \mathrm{~min}$. The cells were incubated overnight at $4{ }^{\circ} \mathrm{C}$ with mouse anti-VE-cadherin, anti- $\beta$-catenin, or Alexa568-conjugated phalloidin (1:1000, Sigma, St. Louis, MO, USA), diluted with PBS, and were incubated at $37^{\circ} \mathrm{C}$ for $1.5 \mathrm{~h}$ with FITC-conjugated goat anti-mouse IgG (1:100, Zhongshan Biotechnology Co. Ltd., Beijing, China) diluted with PBS. The cells after washing were observed with confocal microscope (Zeiss, Munich, Germany).

\subsection{Western Blot}

HUVEC cells treated with SMG or the control condition were lysed with RIPA buffer (150 mM sodium chloride, 1\% Triton X-100, 0.5\% sodium deoxycholate, $0.1 \%$ sodium dodecyl sulfate, $50 \mathrm{mM}$ Tris, $\mathrm{pH}$ 8.0). Aliquots of the protein were separated with $12 \%$ SDS-PAGE and transferred onto PVDF filters. The membranes were blocked with PBS-T containing $5 \%$ milk and then incubated overnight at $4{ }^{\circ} \mathrm{C}$ with primary antibody (Rap1 (Abcam, Cambridge, UK), Rap2 (Santa Cruz, CA, USA), C3G (Santa Cruz, CA, USA), Rap1GAP (Abcam, Cambridge, England), VE-cadherin (Abcam, Cambridge, UK), $\beta$-catenin (Santa Cruz, CA, USA) or GAPDH (Sigma, Shanghai, China), and the PVDF filters were incubated for $1 \mathrm{~h}$ with HRP-conjugated secondary antibody. ECL reaction (Sangon Biotech, Shanghai, China) was used to detect the signals. The relative expressions of detected protein were normalized to amount of GAPDH.

\subsection{GST Pull-Down Assay for Activation of Rap1 and Rap2}

HUVEC cells under SMG treatment or control condition were lysed in Ral buffer (1\% NP-40, 10\% Glycerol, 50 mM Tris- $\mathrm{HCl}$ (pH7.4), $150 \mathrm{mM} \mathrm{NaCl,} 2.5 \mathrm{mM} \mathrm{MgCl}$, $10 \mathrm{mM}$ $\mathrm{NaF}, 1 \mathrm{mM} \mathrm{Na} \mathrm{VO}_{4}, 1 \mu \mathrm{g} / \mathrm{mL}$ Leupeptin, $1 \mu \mathrm{g} / \mathrm{mL}$ Aprotinin, $1 \mathrm{mM}$ PMSF). The cell lysates were centrifuged at $12,000 \mathrm{rpm}$ for $15 \mathrm{~min}$ at $4{ }^{\circ} \mathrm{C}$. The activation of Rap 1 and Rap2 in the supernatant fraction was carried out with GST pull-down assay as described previously [46].

\subsection{Data Analysis}

All the experiments were repeated at least three times. Statistical significance was performed using Student's $t$-test. Differences were considered to be significant at $p<0.05$.

\section{Conclusions}

According to the experimental results obtained, the following conclusions are drawn:

(1) The simulated microgravity effect can affect the expression level and activation state of Rap1 and Rap2 in the cell, and the SMG condition will affect the adhesion and connection of monolayer HUVEC cells. With the extension of the treatment, the strength of the adhesion and connection between the cells would decrease and then recover, reaching the weakest state at $24 \mathrm{~h}$ of SMG.

(2) The simulated microgravity effect has an impact on the expression of four proteins that have an impact on cell adhesion and junction in HUVEC cells: Rap1GAP, C3G, VE-cadherin, and $\beta$-catenin. Under the SMG effect, the expression of Rap1GAP and $\mathrm{C} 3 \mathrm{G}$ proteins would increase and then decrease, while that of VE-cadherin and $\beta$-catenin proteins would decrease and then recover.

(3) Under the simulated microgravity effect, the two proteins Rap1GAP and VE-cadherin would affect the adhesion and connection state of the monolayer HUVEC cells. When the intracellular Rap1GAP was interfered with, the intercellular adhesion would slightly enhance under the SMG effect. When intracellular VE-cadherin was interfered 
with, the adhesion and junction between cells under the effect of SMG would slightly enhance and then recover.

Supplementary Materials: The following are available online at https:/ /www.mdpi.com/article/10.3390/ ijms23020630/s1.

Author Contributions: Conceptualization, J.L. and S.S.; methodology, J.L., S.S., W.G., Y.H., J.W., Y.Z., L.Y. and L.W.; software, S.S., J.L., E.L. and L.W.; validation, J.L. and S.S.; formal analysis, S.S., L.W. and E.L.; investigation, J.L. and S.S.; resources, S.S. and L.W.; data curation, J.L., S.S. and E.L.; writing-original draft preparation, S.S., E.L. and L.W.; writing-review and editing, S.S., E.L. and L.W.; supervision, S.S. and L.W.; project administration, S.S.; funding acquisition, L.W. All authors have read and agreed to the published version of the manuscript.

Funding: This research was supported by the opening foundation of the State Key Laboratory of Space Medicine Fundamentals and Application, Chinese Astronaut Research and Training Center, grant number SMFA20K02.

Institutional Review Board Statement: Not applicable.

Informed Consent Statement: Not applicable.

Data Availability Statement: All data are available in this publication and in the Supplementary Materials.

Acknowledgments: Sincere appreciation to Meiling Yang and Jinchao Kang for valuable help regarding this work.

Conflicts of Interest: The authors declare no conflict of interest.

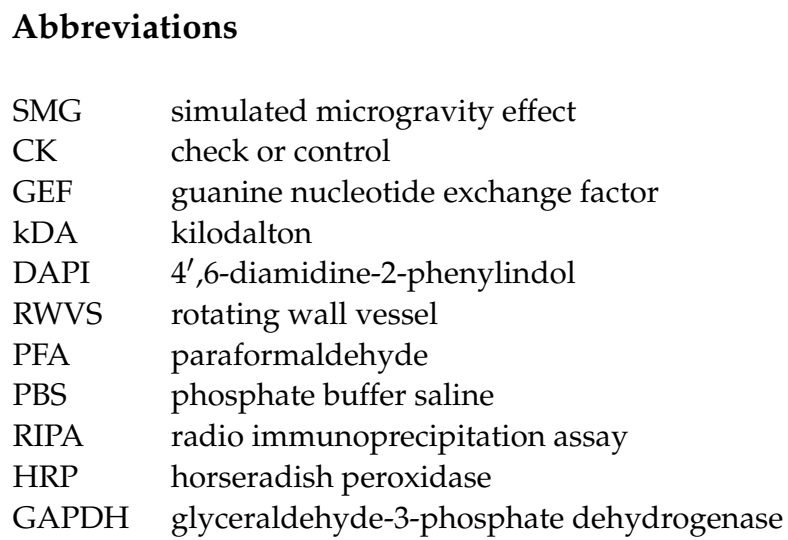

\section{References}

1. Marchio, P.; Guerra-Ojeda, S.; Vila, J.M.; Aldasoro, M.; Victor, V.M.; Mauricio, M.D. Targeting Early Atherosclerosis: A Focus on Oxidative Stress and Inflammation. Oxid. Med. Cell Longev. 2019, 2019, 8563845. [CrossRef] [PubMed]

2. Mehta, D.; Malik, A.B. Signaling mechanisms regulating endothelial permeability. Physiol. Rev. 2006, 86, 279-367. [CrossRef]

3. Alexander, G.P.; Francisco, C.M.A.; Martha, V.A.; Hilda, V.R.; Michael, S. The role of actin-binding proteins in the control of endothelial barrier integrity. Thromb. Haemost. 2015, 113, 20-36.

4. $\quad$ Laura, F.M.; Beatriz, M.R.; Carolina, L.B.; Mariona, G.; Robert, J.C.; Natalia, R.R.; Anaïs, J.; Eva, C.M.; Isabel, C.; Susan, C. Crosstalk between reticular adherens junctions and platelet endothelial cell adhesion molecule-1 regulates endothelial barrier function. Arter. Thromb. Vasc. Biol. 2012, 32, e90-e102.

5. Lampugnani, M.G.; Corada, M.; Caveda, L.; Breviario, F.; Ayalon, O.; Geiger, B.; Dejana, E. The molecular organization of endothelial cell to cell junctions: Differential association of plakoglobin, beta-catenin, and alpha-catenin with vascular endothelial cadherin (VE-cadherin). J. Cell Biol. 1995, 129, 203-217. [CrossRef] [PubMed]

6. Suzuki, S.; Sano, K.; Tanihara, H. Diversity of the cadherin family: Evidence for eight new cadherins in nervous tissue. Cell Regul. 1991, 2, 261-270. [CrossRef]

7. Lampugnani, M.G.; Zanetti, A.; Breviario, F.; Balconi, G.; Orsenigo, F.; Corada, M.; Spagnuolo, R.; Betson, M.; Braga, V.; Dejana, E. VE-cadherin regulates endothelial actin activating Rac and increasing membrane association of Tiam. Mol. Biol. Cell 2002, 13, 1175-1189. [CrossRef] 
8. Iyer, S.; Ferreri, D.M.; DeCocco, N.C.; Minnear, F.L.; Vincent, P.A. VE-cadherin-p120 interaction is required for maintenance of endothelial barrier function. Am. J. Physiol. Lung Cell Mol. Physiol. 2004, 286, L1143-L1153. [CrossRef]

9. Gianfranco, B.; Elisabetta, D. Endothelial cell-to-cell junctions: Molecular organization and role in vascular homeostasis. Physiol. Rev. 2004, 84, 869-901.

10. Hägerling, R.; Hoppe, E.; Dierkes, C.; Stehling, M.; Makinen, T.; Butz, S.; Vestweber, D.; Kiefer, F. Distinct roles of VE-cadherin for development and maintenance of specific lymph vessel beds. EMBO J. 2018, 37, e98271. [CrossRef]

11. Castro Dias, M.; Mapunda, J.A.; Vladymyrov, M.; Engelhardt, B. Structure andjunctional complexes of endothelial, epithelial and glial brain barriers. Int. J. Mol. Sci. 2019, 20, 5372. [CrossRef]

12. Grimsley-Myers, C.M.; Isaacson, R.H.; Cadwell, C.M.; Campos, J.; Hernandes, M.S.; Myers, K.R.; Kowalczyk, A.P. VE-cadherin endocytosis controls vascular integrity and patterning during development. J. Cell Biol. 2020, 219, e201909081. [CrossRef]

13. Chiasson, C.M.; Wittich, K.B.; Vincent, P.A.; Faundez, V.; Kowalczyk, A.P. Kowalczyk P120-Catenin Inhibits VE-Cadherin Internalization through a Rho-independent Mechanism. Mol. Biol. Cell 2009, 20, 1970-1980. [CrossRef]

14. Lisowska, J.; Rödel, C.J.; Manet, S.; Miroshnikova, Y.A.; Boyault, C.; Planus, E.; De Mets, R.; Lee, H.H.; Destaing, O.; Mertani, H.; et al. The CCM1-CCM2 complex controls complementary functions of ROCK1 and ROCK2 that are required for endothelial integrity. J. Cell Sci. 2018, 131, jcs216093. [CrossRef]

15. Cain, R.J.; Vanhaesebroeck, B.; Ridley, A.J. The PI3K p110 $\alpha$ isoform regulates endothelial adherens junctions via Pyk2 and Rac1. J. Cell Biol. 2010, 188, 863-876. [CrossRef]

16. Polakis, P.G.; Rubinfeld, B.; Evans, T.; McCormick, F. Purification of a plasma membrane-associated GTPase-activating protein specific for rap1/Krev-1 from HL60 cells. Proc. Natl. Acad. Sci. USA 1991, 88, 239-243. [CrossRef]

17. Pannekoek, W.-J.; Post, A.; Bos, J.L. Rap1 signaling in endothelial barrier control. Cell Adhes. Migr. 2014, 8, 100-107. [CrossRef] [PubMed]

18. Hewat, E.A.; Durmort, C.; Jacquamet, L.; Concord, E.; Gulino-Debrac, D. Architecture of the VE-cadherin hexamer. Architecture of the VE-cadherin hexamer. J. Mol. Biol. 2007, 365, 744-751. [CrossRef] [PubMed]

19. de Rooij, J.; Zwartkruis, F.J.; Verheijen, M.H.; Cool, R.H.; Nijman, S.M.; Wittinghofer, A.; Bos, J.L. Epac is a Rap1 guaninenucleotide-exchange factor directly activated by cyclic AMP. Nature 1998, 396, 474-477. [CrossRef]

20. van den Berghe, N.; Cool, R.H.; Horn, G.; Wittinghofer, A. Biochemical characterization of C3G: An exchange factor that discriminates between Rap1 and Rap2 and is not inhibited by Rap1A(S17N). Oncogene 1997, 15, 845-850. [CrossRef] [PubMed]

21. Ohba, Y.; Mochizuki, N.; Matsuo, K.; Yamashita, S.; Nakaya, M.; Hashimoto, Y.; Hamaguchi, M.; Kurata, T.; Nagashima, K.; Matsuda, M. Rap2 as a slowly responding molecular switch in the Rap1 signaling cascade. Mol. Cell Biol. 2000, 20, 6074-6083. [CrossRef]

22. Parker, W.H.; Rhea, E.M.; Qu, Z.C.; Hecker, M.R.; May, J.M. Intracellular ascorbate tightens the endothelial permeability barrier through Epac1 and the tubulin cytoskeleton. Am. J. Physiol. Cell Physiol. 2016, 311, C652-C662. [CrossRef] [PubMed]

23. Ohba, Y.; Ikuta, K.; Ogura, A.; Matsuda, J.; Mochizuki, N.; Nagashima, K.; Kurokawa, K.; Mayer, B.J.; Maki, K.; Miyazaki, J.; et al. Requirement for C3G-dependent Rap1 activation for cell adhesion and embryogenesis. EMBO J. 2001, 20, 3333-3341. [CrossRef] [PubMed]

24. Birukova, A.A.; Tian, X.; Tian, Y.; Higginbotham, K.; Birukov, K.G. Rap-afadin axis in control of Rho signaling and endothelial barrier recovery. Mol. Biol. Cell 2013, 24, 2678-2688. [CrossRef] [PubMed]

25. Kitayama, H.; Sugimoto, Y.; Matsuzaki, T.; Ikawa, Y.; Noda, M. A ras-related gene with transformation suppressor activity. Cell 1989, 56, 77-84. [CrossRef]

26. Tsukamoto, N.; Hattori, M.; Yang, H.; Bos, J.L.; Minato, N. Rap1 GTPase-activating protein SPA-1 negatively regulates cell adhesion. J. Biol. Chem. 1999, 274, 18463-18469. [CrossRef] [PubMed]

27. Knox, A.L.; Brown, N.H. Rap1 GTPase regulation of adherens junction positioning and cell adhesion. Science 2002, 295, 1285-1288. [CrossRef]

28. Bonello, T.T.; Perez-Vale, K.Z.; Sumigray, K.D.; Peifer, M. Rap1 acts via multiple mechanisms to position Canoe and adherens junctions and mediate apical-basal polarity establishment. Development 2018, 145, dev157941. [CrossRef]

29. Wittchen, E.S.; Worthylake, R.A.; Kelly, P.; Casey, P.J.; Quilliam, L.A.; Burridge, K. Rap1 GTPase inhibits leukocyte transmigration by promoting endothelial barrier function. J. Biol. Chem. 2005, 280, 11675-11682. [CrossRef]

30. Pizon, V.; Chardin, P.; Lerosey, I.; Olofsson, B.; Tavitian, A. Human cDNAs rap1 and rap2 homologous to the drosophila gene Dras3 encode proteins closely related to ras in the 'effector' region. Oncogene 1988, 3, 201-204.

31. Rubinfeld, B.; Munemitsu, S.; Clark, R.; Conroy, L.; Watt, K.; Crosier, W.J.; McCormick, F.; Polakis, P. Molecular cloning of a GTPase activating protein specific for the Krev-1 protein p21 rap1. Cell 1991, 65, 1033-1042. [CrossRef]

32. Niola, F.; Zhao, X.; Singh, D.; Sullivan, R.; Castano, A.; Verrico, A.; Lasorella, A. Mesenchymal high-grade glioma is maintained by the ID-RAP1 axis. J. Clin. Investig. 2013, 123, 405-417. [CrossRef] [PubMed]

33. Pannekoek, W.J.; Linnemann, J.R.; Brouwer, P.M.; Bos, J.L.; Rehmann, H. Rap1 and Rap2 antagonistically control endothelial barrier resistance. PLoS ONE. 2013, 8, e57903. [CrossRef] [PubMed]

34. Greco, F.; Ciana, A.; Pietra, D.; Balduini, C.; Minetti, G.; Torti, M. Rap2, but not Rap1 GTPase is expressed in human red blood cells and is involved in vesiculation. Biochim. Biophys. Acta 2006, 1763, 330-335. [CrossRef]

35. Huang, H.; Di, J.; Qu, D.; Gao, Z.; Zhang, Y.; Zheng, J. Role of Rap2 and its Downstream Effectors in Tumorigenesis. Anti-Cancer Agents Med. Chem. 2015, 15, 1269-1276. [CrossRef] 
36. Bellone, J.A.; Gifford, P.S.; Nishiyama, N.C.; Hartman, R.E.; Mao, X.W. Long-term effects of simulated microgravity and/or chronic exposure to low-dose gamma radiation on behavior and blood-brain barrier integrity. NPJ Microgravity 2016, $2,16019$. [CrossRef]

37. Kapitonova, M.Y.; Kuznetsov, S.L.; Froemming, G.R.; Muid, S.; Nor-Ashikin, M.N.; Otman, S.; Shahir, A.R.; Nawawi, H. Effects of space mission factors on the morphology and Function of endothelial cells. Bull. Exp. Biol. Med. 2013, 154, 796-801. [CrossRef]

38. Crawford-Young, S.J. Effects of microgravity on cell cytoskeleton and embryogenesis. Int. J. Dev. Biol. 2003, 50, 183-191. [CrossRef]

39. Szulcek, R.; van Bezu, J.; Boonstra, J.; van Loon, J.J.; van Nieuw Amerongen, G.P. Transient intervals of hyper-gravity enhance endothelial barrier integrity: Impact of mechanical and gravitational forces measured electrically. PLoS ONE 2015, 10, e0144269. [CrossRef]

40. Monteiro, A.C.; Luissint, A.-C.; Sumagin, R.; Lai, C.; Vielmuth, F.; Wolf, M.F.; Laur, O.; Reiss, K.; Spindler, V.; Stehle, T.; et al. Trans-dimerization of JAM-A regulates Rap2 and is mediated by a domain that is distinct from the cis-dimerization interface. Mol. Biol. Cell 2014, 25, 1574-1585. [CrossRef]

41. Wehland, M.; Ma, X.; Braun, M.; Hauslage, J.; Hemmersbach, R.; Bauer, J.; Grosse, J.; Infanger, M.; Grimm, D. The impact of altered gravity and vibration on endothelial cells during a parabolic flight. Cell Physiol. Biochem. 2013, 31, 432-451. [CrossRef]

42. Kang, J.C. Study on the Response of Cytoskeleton System to Mechanical Changes. Master's Thesis, Harbin Institute of Technology, Harbin, China, 30 June 2015.

43. Tsygankova, O.M.; Wang, H.; Meinkoth, J.L. Tumor cell migration and invasion are enhanced by depletion of Rap1 GTPaseactivating protein (Rap1GAP). J. Biol. Chem. 2013, 288, 24636-24646. [CrossRef]

44. Ukropec, J.A.; Hollinger, M.K.; Woolkalis, M.J. Regulation of VE-cadherin linkage to the cytoskeleton in endothelial cells exposed to fluid shear stress. Exp. Cell Res. 2002, 273, 240-247. [CrossRef] [PubMed]

45. Tsygankova, O.M.; Ma, C.; Tang, W.; Korch, C.; Feldman, M.D.; Lv, Y.; Brose, M.S.; Meinkoth, J.L. Downregulation of Rap1GAP in human tumor cells alters cell/matrix and cell/cell adhesion. Mol. Cell Biol. 2010, 30, 3262-3274. [CrossRef] [PubMed]

46. Zwartkruis, F.J.; Wolthuis, R.M.; Nabben, N.M.; Franke, B.; Bos, J.L. Extracellular signal-regulated activation of Rap1 fails to interfere in Ras effector signalling. EMBO J. 1998, 17, 5905-5912. [CrossRef] [PubMed]

47. Tan, Y.J.; Yu, J.R.; Nie, J.L.; Wang, C.Y.; Yang, F.; Ding, B.; Gu, Y.; Qu, L.N.; Wang, Y.M.; Li, Y.H. Development of two direction multi-sample cell experimental device for microgravity effects simulation. Space Med. Med. Eng. 2011, 1, 13-16.

48. Shi, S.L.; Li, Q.; Cao, Q.; Diao, Y.; Zhang, Y.; Yue, L.; Wei, L.J. EMT transcription factors are involved in the altered cell adhesion under simulated microgravity effect or overloading by regulation of E-cadherin. Int. J. Mol. Sci. 2020, 21, 1349. [CrossRef] 